\title{
The Application of AHP Model in College Students Education Quality Assessment
}

\author{
Xue Yingzhen ${ }^{1, \text { a }}$ \\ ${ }^{1}$ Xi'an International University, business school, Shaanxi,xian, 70077,china \\ axueyingzhen@xaiu.edu.cn
}

Keywords: AHP Model, College Students, Education Quality, Assessment

\begin{abstract}
AHP is a systematic, hierarchical, multi-criteria decision making method which combines the qualitative and quantitative. In this paper, we study the application of AHP model in college students' education quality assessment, promote the basic principles of students' personnel training quality evaluation and designed the evaluation index system. And this paper pointed out that in order to improve the quality of college students, we must focused on training programs, training measures and the quality evaluation according to the order of importance, only in this way can we provide high-quality talent to the public.
\end{abstract}

\section{Introduction}

With the vigorous development of China's higher education, more and more students into the market. Business for selection of personnel requirements are also increasing, especially in the overall quality of the employer. How effective the overall quality of college students, rational, scientific evaluation, is an important indicator which being quite concerned in enterprises employing assessment, as well as universities in personnel training and promote the overall quality of the educational process faces a very important issue [1].

Analytic Hierarchy Process is the T. L. Saaty Professor at the University of Pittsburgh in the 1970s proposed. It is a combination of qualitative and quantitative, systematic, hierarchical analysis. Features of AHP is in essence after in-depth analysis of complex decision-making problems, influencing factors and internal relations, build a hierarchical model, and then use less quantitative information to the decision-making process of mathematical thinking, so as to solve the multi-target multiple criteria or no structural characteristics of complex decision problems provides an easy approach to decision making. Traditional AHP is to compare the relative importance of the evaluation factors pair wise, then sorted. In practice, since the one-sidedness of human judgment and subjectivity, pair wise comparisons of the results do not necessarily have an objective consistency, therefore, need consistency check if it cannot pass the test with conventional practice is a rough estimate to adjust the judgment matrix. After conventional AHP improved use 0-1 scale method, making it natural to meet the conformance requirements, without consistency check, eliminating the traditional consistency checking and adjustment of the traditional matrix.

\section{The Introduction of AHP}

AHP is a combination of qualitative and quantitative, systematic, hierarchical multi-criteria decision making methods. It can make people's thought processes hierarchical, drill through a variety of related factors of comparative analysis, decision-making, to predict or control the development of things provide quantitative basis. Especially suitable for those complex problems is difficult to completely quantitative analysis, modeling is an effective way to solve such problems [2].

\section{The Basic Principle of AHP}

The measure principle of AHP. Decision-making is a group known solution is selected from the ideal solution, for decision-making model of social and economic system and it is often difficult to 
measure quantitatively. Therefore, the core of AHP decision model is a measure of factors. Analytic Hierarchy Process for Measuring the socio-economic system with the characteristics, providing basic ways to measure decision-making, which uses relative scale pair wise comparison in order to achieve tangible and asexual, quantitative and non-quantitative factors unified measure.

The principle of hierarchical structure. Part or element of a complex problem can be decomposed into several components, namely, goals, constraints guidelines, programs and the like. According to different attributes of these factors intersect grouping complementary level, on a level factors on all or part of the adjacent next level factors play a dominant role in the formation of the layer by layer hierarchical top-down relations of domination.

The ordering principle of AHP. Analytic Hierarchy Process scheduling problem, in essence, is a set of factors pair wise comparison of its importance, the calculation factors measure the relative importance of the issue [3].

\section{The Features of AHP}

Systemic. System requirements analysis to analyze the object thought as a whole, and to distinguish between levels of the system, but the ideological basis and principles of systems analysis AHP is consistent. It requires policy makers to make decisions when problem analysis, analysis of relevant factors associated with each object is to establish system-level hierarchical structure. The structure can clearly reflect the relationship between the relevant factors.

Synthesis. At present a large number of decision-making problems, the factors to be considered by many policy-makers belong to qualitative factors, which cannot be some kind of quantitative scale performance. When AHP decision analysis, be able to issue a comprehensive quantitative and qualitative analysis process and get a clear quantitative conclusion.

Ease. AHP judgment decision-making process is very simple, if complemented by computer and related procedures, the entire calculation process is more convenient and rapid.

Accuracy. AHP that policy makers can provide "satisfactory decision", it can also provide "the best decision." The rich mathematical principles provide a credible basis for the accuracy of AHP. AHP method can also draw policymakers individual or group experience, wisdom, and judgment.

\section{The Basic Steps of AHP}

Applying AHP, substantially according to the following four steps to achieve [4]:

Step 1: analyze the relationship between the various factors in the system and create a system of hierarchical structure. The first step is to construct a hierarchical design method hierarchy. Generally it is divided into overall objective, rule and the program layer. When a level when the content is too complicated, it can be broken down into several levels, such as the overall objective can be broken down into several sub-goals, one criterion can be broken down into several sub-criteria, and so on. Completion of the hierarchy has a tree structure, showing the pyramid.

Step 2: the same level of importance of each element on a layer in a pair wise comparison criterion, construct pair wise comparison judgment matrix. The importance of comparative judgment matrix is dependent on the same factors in the upper layer of each of the factors used in the same. Judgment matrix is usually represented by A, its elements ranging from 1 to 9 , according to the importance of scale value factors.

Step 3: Calculate the judgment matrix element comparison of the relative weights of the criteria, and judgment matrix consistency test.

Step 4: Calculate the total system at all levels and for sorting and sorting. Finally, give a total for the overall goal of the programs of the sort.

\section{The Current Situation and Problems of College Students Education Quality Assessment}

Universities continued enrollment lead to lack of educational resources, the quality of students 
vary greatly and other issues, the teaching process and running a strong impact on order, and competition between social organizations is increasingly reflected in the talent competition, the cultivation of college students It poses a severe challenge. However, China Quality Evaluation System for Training at the heart of University Students has not attracted enough attention. Existing research has focused on the following areas [5]:

The training target and the location of colleges and universities. About training objectives, existing research most of that should be divided into research, application-oriented, complex and innovative talents, all kinds of talents to meet the needs of social division of labor should have the knowledge of the structure and expertise to universities should be based on its own hardware and software Environmental training objectives for a reasonable positioning. Positional Problems of Higher Education universities considered to be generated in a specific historical stage, mainly for convergence and rising, moderate rising to foster convergence and higher education markets and improve school efficiency have some positive effect, but its negative effects cannot be discounted.

The culture process. Previous studies focused on the credit system reform, cooperation between universities, research cooperation and international cooperation. From the development trend, influence the process of internationalization of education, the training process has been extended to the school campus, or even abroad. Heuristic training methods and training process active practice, emphasize the development of high quality innovative talents.

The evaluation of the education quality. Most existing studies evaluate the quality of education that the universities generally include self-evaluation, evaluation and social evaluation of the Government, the literature focused on the overall evaluation of the university's self. The overall framework of education quality assessment system involves evaluation of the teaching process, evaluation of teaching effectiveness and knowledge of talent, ability and quality of evaluation. Teaching quality assurance and monitoring system including the definition of objectives, the establishment of the main teaching quality standards, statistics and measurement, evaluation, feedback, control and other aspects of the work of six.

The reference and comparative of foreign education. Existing research mainly through introducing success stories Talents United States, Britain, Germany and Australia and other countries the way, teaching ideas and methods, targeting, and other aspects of quality assessment, analysis of national educational development. While the general requirements in Western countries undergraduate curriculum, curriculum design, structure, and timing were compared, analyzed their respective advantages, features, and then put forward countermeasures and suggestions for the reform of higher education.

\section{The Analysis of Cultivation Quality Evaluation System of College Students}

At present, China on Undergraduate Education Quality Evaluation Personnel Reliability and validity have some problems, such as target setting is not comprehensive, standard-setting is not accurate, so that the quality of the evaluation process cannot meet the needs of management needs and community colleges to universities evaluation. As used herein, AHP, after building the quality of talent evaluation Undergraduate, using AHP to determine the appropriate weights for each index expanded evaluation, may reasonably dispose of relationships between decision-making factors, so that the evaluation results sufficient theoretical basis of various indicators, scientific and rational . On terms of individual universities, colleges and universities may Talents expand multi-factor evaluation, making universities a comprehensive understanding of their own, understand the advantages and shortcomings, clearly the direction of future progress; also via several different universities personnel training quality lateral alignment, to make public departments and the public on the quality of each undergraduate university personnel training and judgment to make just the right sort, provide a reference for the formulation and revision of relevant policies.

According to the target of Undergraduate Talents, basic requirements and characteristics, combined with professional disciplines characteristics, the cornerstone of a comprehensive study on the constituent elements of university talent training quality expand inductive analysis, consultation 
with colleges and universities, students, teachers and potential employers the cornerstone of views, the main aspects of extraction, to construct a reasonable objective scientific evaluation system. Expand the scientific classification of the indicators and to build a hierarchy ladder, clear connotation of the evaluation, the evaluation criteria and evaluation level. In a multi-level, comprehensive consultation on the cornerstone of the views of the stakeholders, AHP measure the weight of each evaluation index weights

\section{Conclusion}

In the quality evaluation of college students, the personnel training quality is affected by multiple factors. Using the AHP to evaluate and analysis personnel training quality, we are able to grasp the main factors to establish hierarchy, make the complicated issues to be simple, reflect the quality of personnel training true and reliable, help to clear the future direction of personnel training, make the effective long-term development planning and personnel training, promoting innovation efforts of students personnel training to meet the needs of socio-economic development.

\section{Acknowledgement}

2015 Scientific Research Program Funded by Shaanxi Provincial Education Department(Program NO:15JK2134); 2015 higher education teaching reform project Funded by Xi'an International University (project number: 2015B04).

\section{References}

[1] L. Jin, The considerations on the quality evaluation system of higher education, J. Baoji College. 44 (2010) 146-147.

[2] Y. Wu, The quality evaluation system of Japanese higher education, J. China Higher Education Research. 18(2005) 55-57.

[3] R.Wang, The quality evaluation system of the undergraduates' training, J. Value Engineering, 32(2012) 189-190.

[4] X.F.Liu, The research on performance evaluation summary of Russia higher education expenditure, J, Central South University, 4(2007) 38-40.

[5] Y.Y.Hong, The AHP, GEM and its comprehensive algorithm, J. China Management Science, 9(2013) 142-150. 